\title{
In vitro differentiation of rat embryonic stem cells into functional cardiomyocytes
}

Nan $\mathrm{Cao}^{1, *}$, Jing $\mathrm{Liao}^{2,{ }^{*}}$, Zumei Liu ${ }^{1}$, Wenmin Zhu ${ }^{1}$, Jia Wang ${ }^{1}$, Lijun $\mathrm{Liu}^{5}$, Lili Yu ${ }^{5}$, Ping Xu ${ }^{5}$, Chun Cui ${ }^{2}$, Lei $\mathrm{Xiao}^{2}$, Huang-Tian Yang ${ }^{1,3,4}$

${ }^{I}$ Key Laboratory of Stem Cell Biology, Institute of Health Sciences, Shanghai Institutes for Biological Sciences (SIBS), Chinese Academy of Sciences (CAS) \& Shanghai Jiao Tong University School of Medicine (SJTUSM), SIBS, CAS, Shanghai 200025, China; ${ }^{2}$ Laboratory of Molecular Cell Biology, Institute of Biochemistry and Cell Biology, Cell Bank, Stem Cell Bank, SIBS, CAS, Shanghai 200032, China; ${ }^{3}$ Shanghai Key Laboratory of Vascular Biology, Ruijin Hospital, SJTUSM, Shanghai 200025, China; ${ }^{4}$ Shanghai Stem Cell Institute, SJTUSM, Shanghai 200025, China; ${ }^{5}$ Shanghai Laboratory Animal Center (SLAC), CAS, Shanghai 200032, China

The recent breakthrough in the generation of rat embryonic stem cells (rESCs) opens the door to application of gene targeting to create models for the study of human diseases. In addition, the in vitro differentiation system from rESCs into derivatives of three germ layers will serve as a powerful tool and resource for the investigation of mammalian development, cell function, tissue repair, and drug discovery. However, these uses have been limited by the difficulty of in vitro differentiation. The aims of this study were to establish an in vitro differentiation system from rESCs and to investigate whether rESCs are capable of forming terminal-differentiated cardiomyocytes. Using newly established rESCs, we found that embryoid body (EB)-based method used in mouse ESC (mESC) differentiation failed to work for the serum-free cultivated rESCs. We then developed a protocol by combination of three chemical inhibitors and feeder-conditioned medium. Under this condition, rESCs formed EBs, propagated and differentiated into three embryonic germ layers. Moreover, rESC-formed EBs could differentiate into spontaneously beating cardiomyocytes after plating. Analyses of molecular, structural, and functional properties revealed that rESC-derived cardiomyocytes were similar to those derived from fetal rat hearts and mESCs. In conclusion, we successfully developed an in vitro differentiation system for rESCs through which functional myocytes were generated and displayed phenotypes of rat fetal cardiomyocytes. This unique cellular system will provide a new approach to study the early development and cardiac function, and serve as an important tool in pharmacological testing and cell therapy.

Keywords: rat ES cells; differentiation; cardiomyocytes

Cell Research (2011) 21:1316-1331. doi:10.1038/cr.2011.48; published online 22 March 2011

\section{Introduction}

The laboratory rat (Rattus norvegicus) is the first mammalian species to be domesticated for scientific research. It has been widely used in physiological, phar-

\footnotetext{
*These two authors contributed equally to this work. Correspondence: Huang-Tian Yang ${ }^{\mathrm{a}}$, Lei Xiao ${ }^{\mathrm{b}}$

${ }^{\mathrm{a}} \mathrm{Tel} / \mathrm{Fax}:+86-21-63852593$

E-mail: htyang@sibs.ac.cn

${ }^{\mathrm{b}}$ Tel: +86-21-54921386; Fax: +86-21-54921388

E-mail: leixiao@sibs.ac.cn

Received 6 September 2010; revised 16 December 2010; accepted 20 January 2011; published online 22 March 2011
}

macological, and toxicological studies for over 150 years because it is of a suitable size and more relevant to humans than mice in both physiological regulation and pharmacological responses [1-3]. Accordingly, the physiology of the rat has been studied extensively, including the cardiovascular system [4]. Moreover, rat experimental models of various human diseases, such as heart failure, hypertension, diabetes, and neurological disorders, have already been well established and widely used in scientific studies and drug discovery for scientific and economic reasons $[2,4,5]$. However, the use of rat genetic models has been restricted because of the lack of rat embryonic stem cells (rESCs).

This situation has now been changed since Austin 
Smith and Qi-Long Ying and their colleagues $[6,7]$ successfully generated authentic chimerable and germlinecompetent rESCs by a combination of the serum-free culture system and specific signal inhibitors in 2008. Since then, several groups have subsequently established rESC lines with similar approaches [8-12]. This important breakthrough will allow us to apply the gene-targeting technique routinely accomplished in mice to create rat models for the study of human diseases that may illuminate the possibility of rats to be a more extensively used scientific model [13]. In fact, rESCs were successfully used to knock out the P53 gene in rats recently [14]. In addition, ESCs possess the unique property to differentiate into various somatic cell types from three germ layers in vitro [15-17]. Therefore, an in vitro differentiation system of rESCs will facilitate the analysis of early development and gene function. It will also provide an optimal model for testing the efficacy and safety of cell transplantation and an opportunity for screening new compounds in a cell-based system.

The unique culture system for rESCs is based on a combination of serum-free conditions and inhibitors of mitogen-activated protein kinase (MEK) and glycogen synthase kinase 3 (GSK3) or plus an inhibitor of fibroblast growth factor (FGF). This culture system has been proven to be critical for the derivation and maintenance of rESCs $[6,7]$. Although rESCs cultivated under such conditions are capable of producing teratomas and contribute to chimeras, these cells are extremely difficult to differentiate in vitro $[7,11]$. Currently, little is known on how to stably induce these cells to differentiate in vitro. Unlike mouse ESCs (mESCs), rESCs die quickly when initiating differentiation in vitro by forming threedimensional aggregates called embryoid bodies (EBs), a classical method to induce ESC differentiation on a schedule similar to that in the embryo $[15,18]$. By using modified EB protocols containing three inhibitors (3i, FGF inhibitor: SU5402, MEK inhibitor: PD184352, and GSK3 inhibitor: CHIR99021) for the first 2 days of differentiation, and then incubating with fetal bovine serum (FBS)-conditioned medium collected from feeders to instigate EB formation, Li et al. [7] induced differentiation of rESCs into derivatives of the three germ layers in vitro. However, the efficiency of EB formation from rESCs was much lower compared with that from mESCs. In addition, detailed investigations of the differentiation of rESC derivatives and functional characteristics of rESC-derived cardiomyocytes (rESCMs) have not yet been reported. To address these essential issues for in vitro differentiation of rESCs and usage of rESC derivatives, in the present study we aimed to: (i) establish a stable in vitro differentiation system for rESCs through which rESCs could differentiate into three germ layers and generate functional cardiomyocytes; (ii) investigate the molecular and structural properties of rESCMs; and (iii) characterize the functional phenotype of rESCMs in direct comparison with the rat fetal cardiomyocytes (rFCMs). The newly established in vitro differentiation system of rESCs, in conjunction with a solid foundation of rat in physiological and behavioral experiments, will not only close the gap between the rat and mouse ESCs as an in vitro study model of choice but also provide an important resource for cardiac functional studies, pharmacological testing, and cell therapy.

\section{Results}

\section{Derivation and characterization of $r E S C s$}

To derive rat pluripotent stem cells, we plated E4.5 rat blastocysts from an inbred strain Dark Agouti (DA) onto mitomycin-inactivated mouse embryonic fibroblast (MEF) feeder layers as described previously $[6,7]$. The derived undifferentiated rESC lines DA8-16 and DA8-21 were routinely maintained on mouse embryonic fibroblast (MEF) feeder layers in serum-free N2B27 medium supplemented with MEK and GSK3 selective inhibitors (2i), $0.4 \mu \mathrm{M}$ PD0325901, and $3 \mu \mathrm{M}$ CHIR99021, as described previously $[6,7]$. Under such conditions, cells formed round and compact colonies with typical undifferentiated stem cell-like morphology (Figure 1A, a, c) and had high alkaline phosphatase (ALP) activity (Figure 1A, e), similar to mESCs maintained in serum and leukemia inhibitory factor (LIF) (Figure 1A, b, d, f). Furthermore, reverse transcription (RT)-PCR analyses showed that the rESCs expressed the key pluripotent ESC markers found in mESCs: octamer-binding factor 4 (Oct4), Nanog homeobox (Nanog), SRY-box containing gene 2 (Sox2), and reduced expression protein $1(\operatorname{Rex} 1)$ (Figure 1B). Immunostaining results further confirmed the expression of the stem-cell markers Oct4, Nanog, and stage-specific embryonic antigen 1 (SSEA1) in both rat and mouse ESCs (Figure 1A, g-1). The analysis of doubling time demonstrated a comparable proliferation rate between rat and mouse ESCs (Figure 1C). To test the pluripotency of rESCs in vivo, the rESCs were injected intramuscularly into two non-obese diabetic/severe combined immune-deficient (NOD/SCID) mice. After 30 days, macroscopic tissue masses, varying in diameter size from 1.5 to $2 \mathrm{~cm}$, were formed at the site of injection in two injected mice. Histological analyses showed that the tumor contained multiple differentiated cell types and tissues of three germ layers (Figure 1D, a) including cartilage (mesoderm), primitive renal cells (endoderm), and pigmentary epithelium (ectoderm) (Figure 1D, b-d). 
A
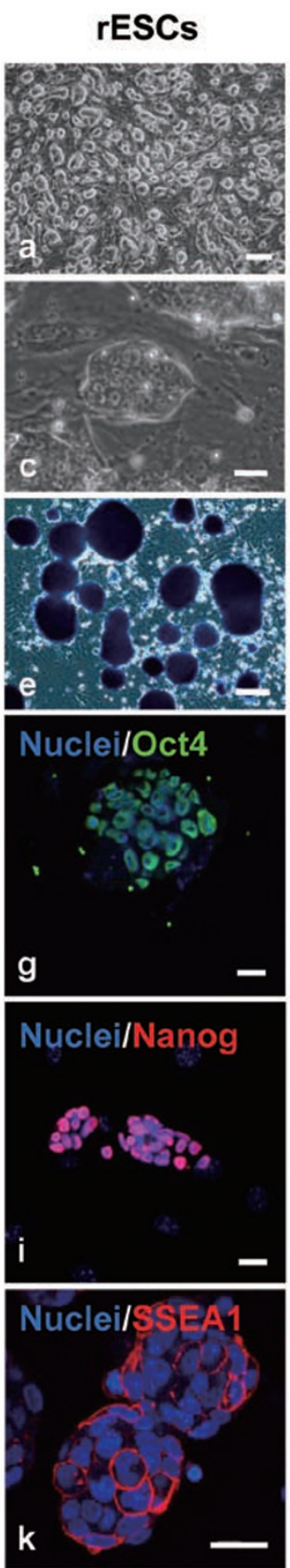

mESCs
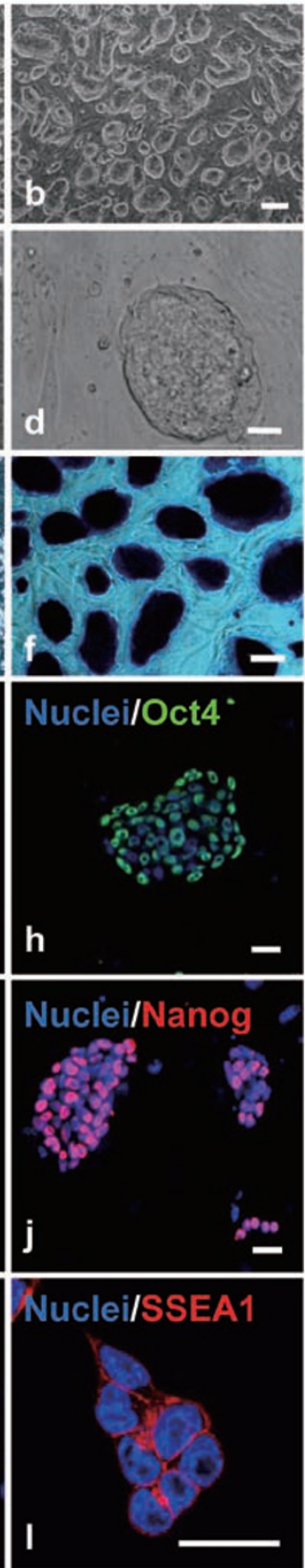

B



C



D

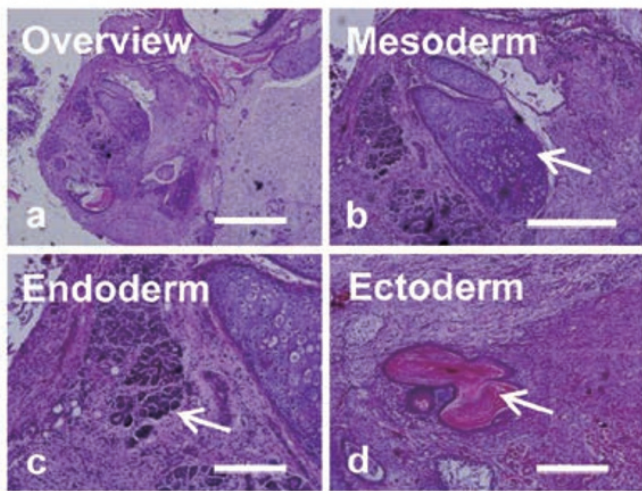

E

DA8-16

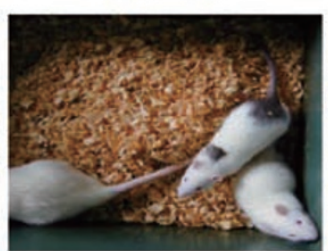

DA8-21



Figure 1 Characteristics of rESCs maintained in 2i conditions. (A) Undifferentiated rESCs (a, c, e, g, i, k) and mESCs (b, d, f, h, j, I). (a-d) Phase contrast images show undifferentiated rESC and mESC colonies on mitotically inactivated MEFs. (e, f) Colonies were positive for ALP staining. (g-l) Colonies were immunopositive for pluripotency markers Oct4 (g, h), Nanog (i, j), and SSEA-1 (k, I). Scale bars $=200 \mu \mathrm{m}(\mathrm{a}, \mathrm{b}), 100 \mu \mathrm{m}$ (e, f), and $20 \mu \mathrm{m}$ (c, d, g-l). Nuclei were counterstained with Hoechst 33258 (blue). (B) RT-PCR analysis of pluripotency marker expressions in rESCs compared with mESCs. Gapdh indicates glyceraldehyde-3-phosphate dehydrogenase, housekeeping gene. (C) Comparison of doubling time between rESCs and mESCs; $n$ = 3. (D) Hematoxylin and eosin staining of histological sections of teratoma derived from rESC line DA8-16. Teratoma (a) was composed of various types of tissue: cartilage (mesoderm, b), primitive renal cells (endoderm, c), and pigmentary epithelium (ectoderm, d). Scale bars $=1 \mathrm{~mm}(\mathrm{a}), 500 \mu \mathrm{m}$ (b), and $200 \mu \mathrm{m}$ (c, d). (E) Generation of live adult coat-color chimeras from both the established rESC lines. Agouti coat-color indicates the donor rESCs of DA strain. MEF, mouse embryonic fibroblast; rESCs, rat embryonic stem cells; mESCs, mouse embryonic stem cells. 
To further prove the pluripotency of these rESC lines, we examined the potential of rESC lines DA8-16 and DA821 to contribute to postnatal chimeras. Mixed agouti- and albino-coloring chimeric animals were obtained from injections of both DA cell lines into albino SD blastocysts (Figure 1E). Four coat-color chimeras, one from DA816 and three from DA8-21, were born and all developed into healthy adults. Taken together, these data confirm that undifferentiated rESCs are similar to mESCs in both morphology and molecular characteristics, and that they are capable of producing teratomas and are competent for mature multi-lineage differentiation in vivo.

Specific environment is required for efficient formation of EBs from $r E S C s$

Next, we examined in vitro differentiation potential of rESCs and explored the possibility of establishing a stable in vitro differentiation system. In vitro differentiation of ESCs normally requires an initial step to allow ESCs to grow in suspension and form three-dimensional aggregates, known as EBs, which resemble early post-implantation embryos and differentiate into derivatives of all three germ layers, including cardiomyocytes [15, 19-21]. Therefore, we applied this classical EB-based method by using a standard hanging-drop technique $[18,19]$ to initiate differentiation of rESCs. First, we used a standard mESC differentiation medium (DM) containing $10 \%$ FBS as reported previously $[18,22]$. However, after 2 days of incubation, the majority of rESCs died instead of forming EBs (Figure 2A, a), suggesting that the presence of $2 \mathrm{i}$ and factors secreted from the MEF cells appear to be critical for the survival and propagation of rESCs. We then modified the standard EB-based protocol by replacing the DM with conditioned medium (CM) collected from MEF cell cultivation. Under this condition, a few rESCs survived and formed small and irregular aggregates (Figure 2A, b). When 2i were further supplemented into the $\mathrm{CM}$ at a quarter of the original concentration $(0.1 \mu \mathrm{M}$ PD0325901 and $0.75 \mu \mathrm{M}$ CHIR99021), larger and more regular EBs formed (Figure 2A, c). However, the aggregation efficiency remained low with many dead cells. It has been reported that Y27632, a selective Rhoassociated kinase inhibitor, could protect human ESCs from dissociation-induced apoptosis and facilitate their differentiation [23]. We thus tested whether Y27632 could further improve the survival of rESCs and increase the efficiency of EB formation. By the addition of 10 $\mu \mathrm{M}$ Y 27632 during the first two days of differentiation, rESCs formed EBs even when cultivated with the DM (Figure 2A, d), generated more regular EBs in the CM (Figure 2A, e), and formed large and homogeneous EBs similar to typical ones from mESCs [20] in CM supple- mented with $2 \mathrm{i}$ (Figure 2A, f), which we defined as rat EB formation medium (rEFM). Meanwhile, to select a suitable concentration of $2 \mathrm{i}$ in the rEFM, we performed systematic experiments to test the effect of $2 \mathrm{i}$ at various concentrations on EB formation. The analysis confirmed that a quarter of the original $2 \mathrm{i}$ concentration was a minimum requirement for forming well-shaped EBs in rEFM (Supplementary information, Figure S1A) and for inducing the highest cardiac-differentiation potential among the concentrations we tested (Supplementary information, Figure S2). Therefore, we used $2 \mathrm{i}$ at a quarter of the original concentration, i.e., 0.1 $\mu \mathrm{M}$ PD0325901 and $0.75 \mu \mathrm{M}$ CHIR99021, and $10 \mu \mathrm{M}$ Y27632 in rEFM and obtained regular EBs as shown in Figure 2A, $\mathrm{f}$.

To determine the apoptosis and necrosis status of the EBs formed under representative conditions, we quantified the viability of cells after 2 days of EB formation. Annexin V (an early apoptosis marker)-propidium iodide (PI) double-staining analyses showed that a small portion $(<40 \%)$ of the rESCs were alive when using the standard mouse DM (Figure 2B). By contrast, the percentage of living cells significantly increased to about $75 \%$ when the EBs of rESCs were formed in the rEFM (Figure 2B).

Next, we tested whether the EBs generated in this specific environment could maintain the in vitro differentiation potential. When persistently cultivated in rEFM during the entire differentiation period, the EBs generated from rESCs retained a typical undifferentiated morphology without the development of visible outgrowth area within 15 days of differentiation (Supplementary information, Figure S1B, a), suggesting the long-term presence of $2 \mathrm{i}$ and Y27632 might impair the differentiation of rESCs. We then examined whether the differentiation process could be improved by the removal of signal inhibitors immediately after EB formation. When day2 EBs generated in rEFM were directly transferred into DM for further suspension cultivation, lots of dead cells appeared at day 4, however, a few EBs persistently grew and maintained healthy morphology at day 6 (Supplementary information, Figure S1B, b-d). These results demonstrate that acute alterations of culture environment might impair the viability of rESCs and thereby prohibit their further differentiation. We thus developed a moderately advancing protocol by sequential removal of Y27632, 2i and CM as shown in Figure 2C. With this protocol, homogeneous EBs were formed at day 2 and retained a clear 3D structure during subsequent 4 days of suspension cultivation (Figure 2D). After plating day-6 EBs onto matrigel-coated dishes in the DM for adherent culture, differentiated specialized cells developed in the outgrowth area of EBs and the area increased upon differentiation from day 7 to day 21 (Figure 2D). RT-PCR 


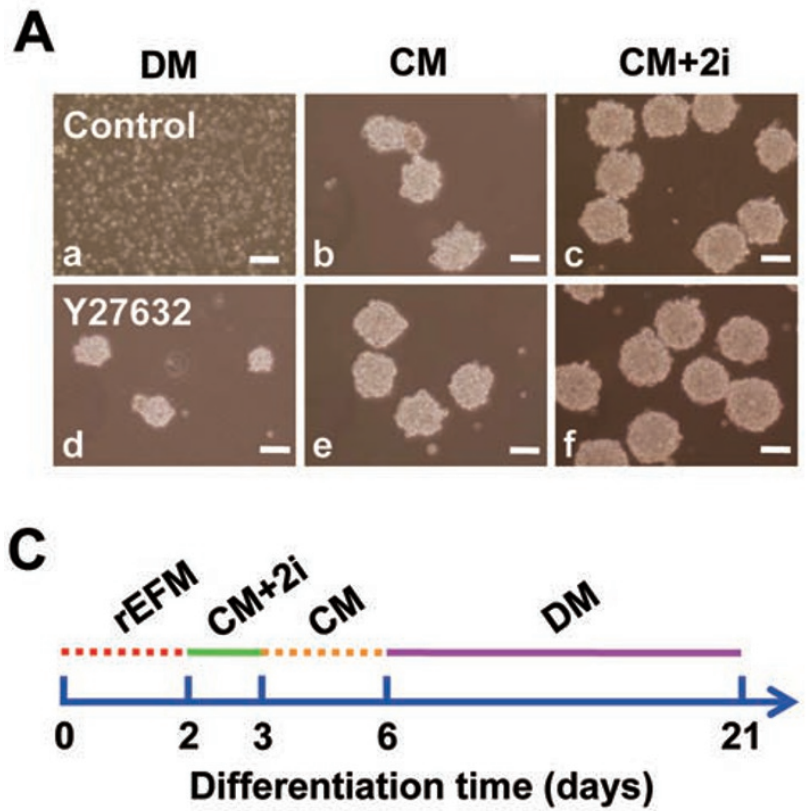

B

D
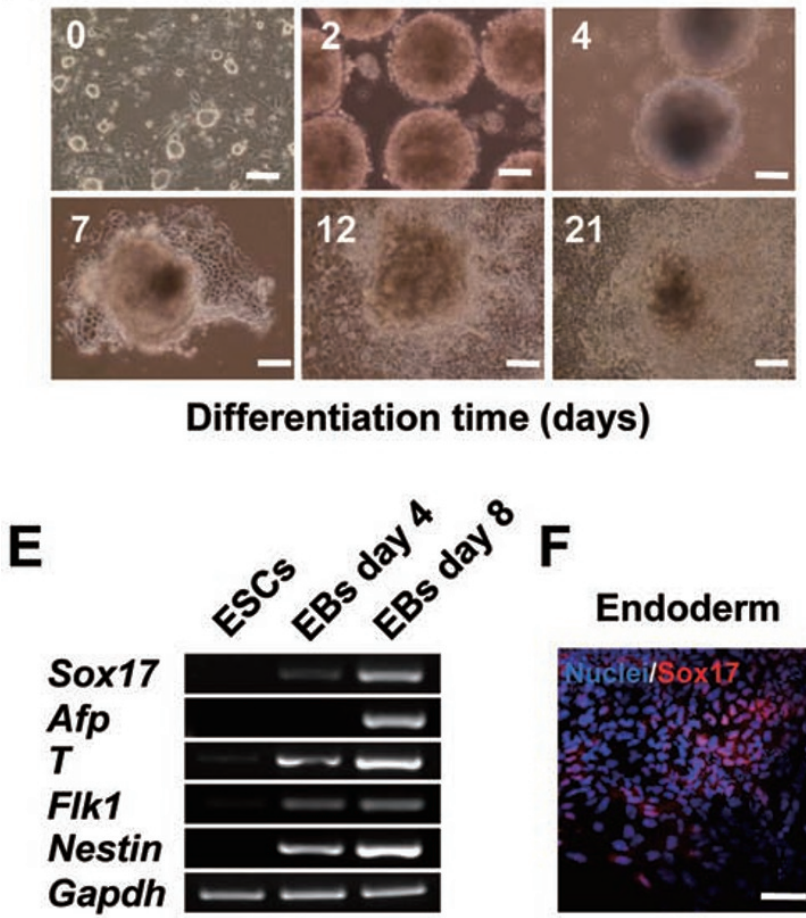

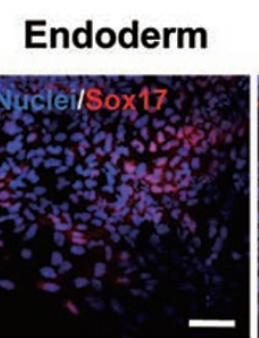


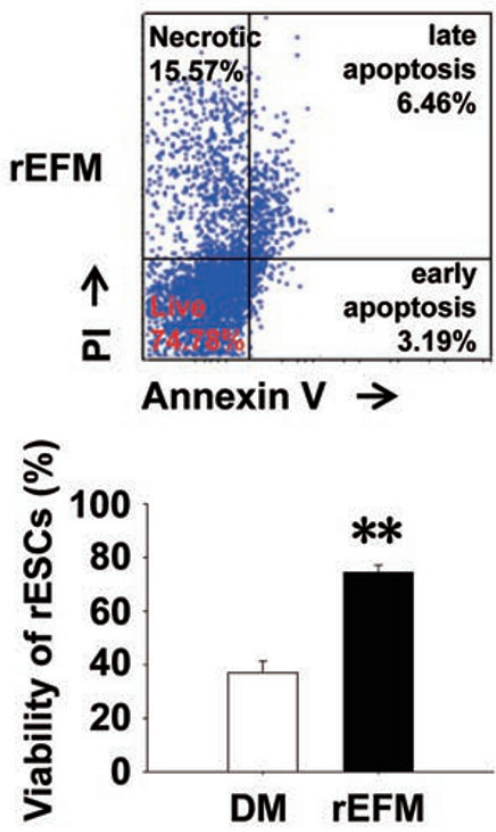

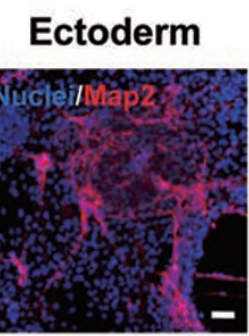

Figure 2 Conditioned medium (CM) with 2i and Y27632 improves the EB formation from rESCs. (A) Phase-contrast images of day-2 EBs formed from rESCs in differentiation medium (DM, a, d), CM (b,e), and CM plus 2i (0.1 $\mu$ M PD0325901 and 0.75 $\mu \mathrm{M}$ CHIR99021, c, f) with and without $10 \mu \mathrm{M}$ of Y27632. Scale bars $=100 \mu \mathrm{m}$. (B) Representative (upper panel) and averaged (lower panel) flow cytometry analysis of apoptosis and necrosis in day-2 differentiating rESCs from the EBs formed in $\mathrm{DM}$ and rEFM based on Annexin V ( $x$ axis) and PI ( $y$ axis) staining. Results were quantified from three independent assays. $* * P<0.01$ vs the DM group. (C) Schematic diagram of the differentiation protocol used. (D) Phase-contrast images of differentiating rESC-derived EBs during differentiation day 0 to day 21. Scale bars $=100 \mu \mathrm{m}$. (E) RT-PCR analysis of germ layer marker expressions in rESCs: days 4 and 8, EBs formed in rEFM. Gapdh, housekeeping gene. (F) Immunofluorescence staining of rESC derivatives on day 12 of differentiation revealed expression of endodermal marker, Sox 17, mesodermal marker, Flk1, and ectodermal marker, Map2. Nuclei were stained with Hoechst 33258 (blue). Scale bars $=50 \mu \mathrm{m}$. rEFM, rat EB formation medium. 
analyses showed that the EBs cultivated in this system expressed lineage commitment markers of all three germ layers including SRY-box containing gene 17 (Sox17) and alpha fetoprotein (Afp, endoderm), brachyury $(T)$ and fetal liver kinase 1 (Flk1, mesoderm), and Nestin (ectoderm) during the differentiation process, which were not expressed in the undifferentiated rESCs (Figure 2E). The analysis of immunofluorescence staining further confirmed that the EBs contained cells positive for endoderm marker, Sox17, mesoderm marker, Flk1, and ectoderm marker, microtubule-associated protein 2 (Map2) (Figure 2F). These results demonstrate that the efficient formation of EBs from rESCs requires a specific environment in which rESCs can survive and differentiate into all three germ layers in vitro.

\section{Differentiation of rESCs into spontaneous beating car- diomyocytes}

ESCs are able to develop into specialized somatic cell types in vitro. One of the most impressive phenotypes among them is the development of spontaneously beating cardiomyocytes $[19,24]$. We thus examined the potential for development of active cardiomyocytes as an example of whether the rESCs cultivated in our established system could differentiate into somatic cells in vitro. Cardiac differentiation was confirmed by the appearance of spontaneously contracting clusters, typical characteristics of cardiomyocytes $[19,24]$ in day-9 EBs at an initial density of 3000 cells per EB (Figure 3A). The percentage of rESC-derived EBs with beating clusters increased gradually and reached the peak at differentiation day 15 in both cell lines DA8-16 and DA8-21 (Figure 3A and Supplementary information, Movies S1 and S2). Because cardiac differentiation of rESCs is also influenced by the initial cell number for EB formation, we compared the initial cell number for EB formation from 500, 1000,2000 , $3000,4000,5000$, to 10000 cells per EB and 3000 cells per EB generated highest percentages of EBs with beating clusters in both cell lines (Figure 3B). The cardiac induction efficiency of this protocol was further evaluated by analysis of cells expressing cardiac troponin $\mathrm{T}$ (cTnT) with flow cytometry. At differentiation day 15, among the total population $6.6 \% \pm 2.0 \%$ cells were $\mathrm{cTnT}$ positive cardiomyocytes in cell line DA8-16 and a comparable efficiency for cardiomyocyte differentiation was observed in cell line DA8-21 (Figure 3C).

Cardiogenesis of ESCs is a well-organized process characterized by ordinal expression of lineage commitment markers and sequenced induction of mesoderm, cardiac precursors, and cardiomyocytes [19, 25-28]. To further characterize the EB-differentiation process, we examined the expression patterns of genes associated with the pluripotent ESC phenotype or with the specific lineage commitment achieved during EB differentiation by RT-PCR analysis. The expression levels of key transcripts Oct4, Nanog, and Rex1 for pluripotent ESCs were high in undifferentiated rESCs, greatly decreased from day 9 , and became faint at differentiation day 21 (Figure 3D). Meanwhile, the expression of mesodermal markers, brachyury and Flk1, as well as cardiac-precursor markers, GATA-binding protein 4 (Gata4), GATA-binding protein 6 (Gata6), NK2 transcription factor-related locus 5 (Nkx2.5), T-box transcription factor 5 (Tbx5), and LIMhomeobox transcription factor islet-1 (Isl1), was detected from differentiation day 4 to day 6 and their expression levels increased with the time of EB differentiation (Figure 3D). Moreover, transcripts of cardiomyocyte markers, myosin regulatory light chain 2 (Myl2) and 7 (Myl7), myosin heavy chain 6 (Myh6), cardiac muscle troponin $\mathrm{T}$ (Tnnt2), and gap junction protein alpha 1 (Gjal), were present from differentiation day 9 and their levels enhanced upon further differentiation (Figure 3D). Concomitant with the expression of these cardiacspecific genes, transcripts coding for major sarcolemmal or sarcoplasmic reticulum (SR) $\mathrm{Ca}^{2+}$-handling proteins, calcium channel alpha 1A subunit (Cacnala), solute carrier family 8 member 1 (Slc8a1), and ryanodine receptor type 2 (Ryr2) also were present from differentiation day 9 and increased upon further differentiation. By contrast, the transcript of inositol 1, 4, 5-triphosphate receptor 2 (Itpr2), an SR $\mathrm{Ca}^{2+}$ release-related gene, was observed in undifferentiated and differentiating rESCs during all time points examined (Figure 3D). The expression patterns of the key pluripotency markers and core cardiac genes during rESC differentiation were further confirmed by quantitative RT-PCR (Q-PCR) analyses. Pluripotent ESC markers, Oct4, Nanog, and Rexl, were significantly downregulated in EBs at differentiation day 21 compared with those in undifferentiated rESCs, whereas robust increases in the expression of the core cardiac transcripts including Gata4, Nkx2.5, Tbx5, Tnnt2, Myh6, and Myl2 were detected in day-21 EBs compared with those in undifferentiated rESCs (Figure 3E). Thus, rESCs can differentiate into spontaneously beating cardiomyocytes in vitro with a well-regulated developmental process as reflected by the expression of cardiac $\mathrm{Ca}^{2+}$-handling proteins and myofilament components by using the modified EB-based method.

rESC-derived cardiomyocytes exhibit typical cross-striated muscle filaments

To further confirm the formation of sarcomere, which is essential for the occurrence of contraction, we compared the expression of specific myofilamental protein 

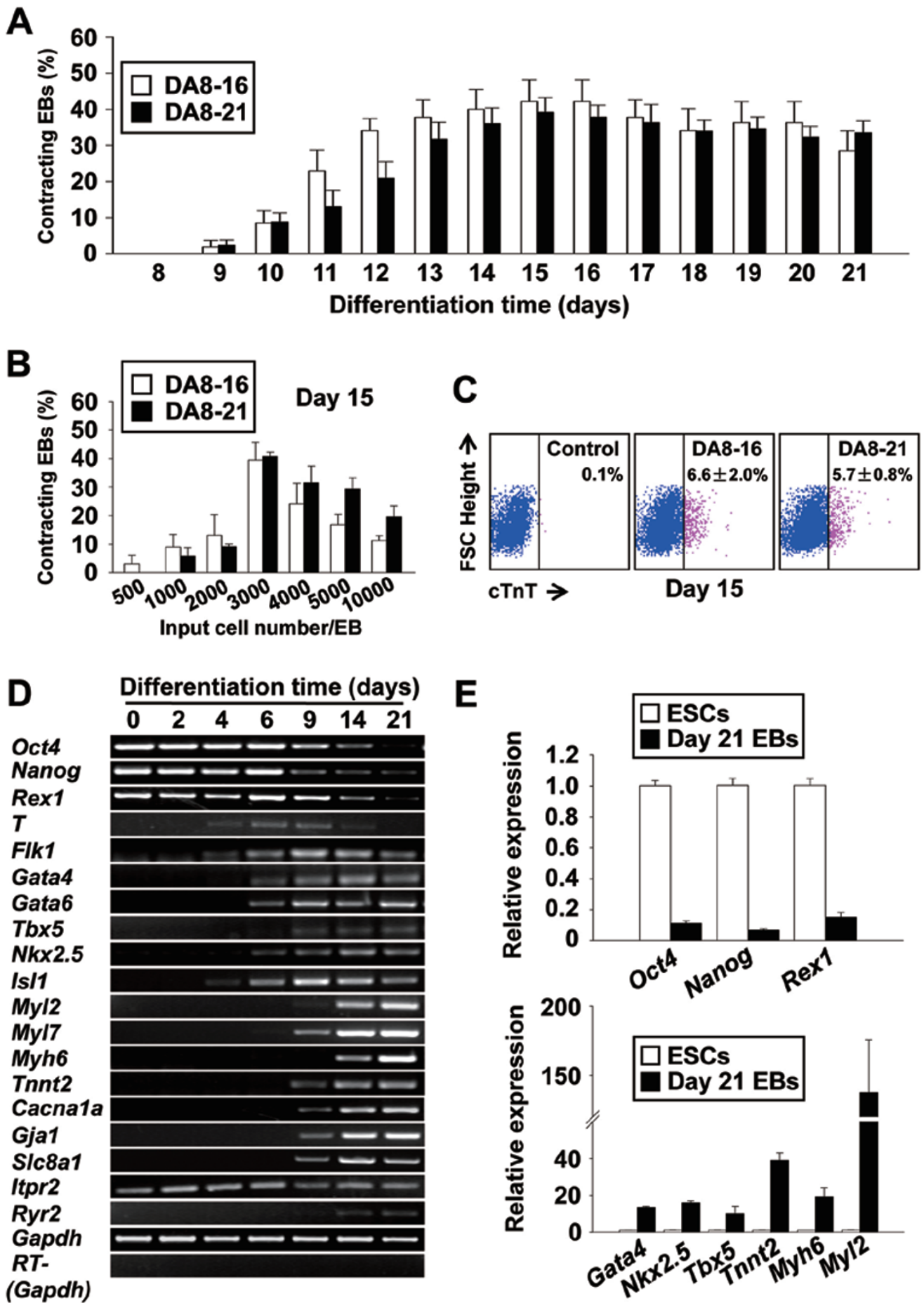

Figure 3 In vitro differentiation of the rESCs induces expression of mesoderm, cardiac progenitor markers, cardiomyocyte markers, and development of contracting EBs over time. (A) The percentage of EBs with contracting areas during differentiation. A total of 222 EBs from clone DA8-16 and 292 EBs from clone DA8-21 were counted from three independent experiments. (B) Identification of the optimal input cell number in each EB for cardiac differentiation of rESCs. Data were analyzed from three independent experiments. (C) Flow cytometry analysis showing the percentage of cardiomyocytes in the total population derived from both rESC lines. Results were quantified at differentiation day 15 from three independent assays. (D) RT-PCR analysis showing the downregulation of pluripotency marker expressions and the upregulation of mesoderm, cardiac progenitor, and cardiac marker expressions. (E) Q-PCR analysis of gene expression levels for pluripotency (upper panel), cardiac progenitor and cardiomyocyte markers (lower panel) in undifferentiated rESC and in day-21 EBs. Data were collected from three independent experiments. 
markers $\alpha$-actinin, cTnT, and cardiac myosin heavy chain (cMHC) among the rESCMs, rFCMs, and cardiomyocytes derived from mESCs (mESCMs). Cells that were positive for $\alpha$-actinin, cTnT, and cMHC were detected at differentiation day 18 of rESC cultures (Figure 4). Immunofluorescence analyses revealed clear striated myofilaments with well-organized cross-striation in the rESCMs (Figure $4 \mathrm{a}, \mathrm{d}, \mathrm{g}$ ) that were comparable with that in timematched rFCMs (Figure $4 \mathrm{~b}, \mathrm{e}, \mathrm{h}$ ) and mESCMs (Figure $4 \mathrm{c}, \mathrm{f}, \mathrm{i})$. Expression of the cardiac-specific transcription factor, myocyte-enhancer factor 2C (Mef2c), was also observed in cMHC-positive cardiomyocytes from each source (Figure 4A, g-i). These data demonstrate that cardiomyocytes derived from rESCs possess a typical sarcomeric structure.

Electrophysiological studies reveal all three major types of cardiomyocytes derived from $r E S C S$

In the next batch of experiments, we assessed the functional integrity of the rESCMs. We first recorded
rESCMs
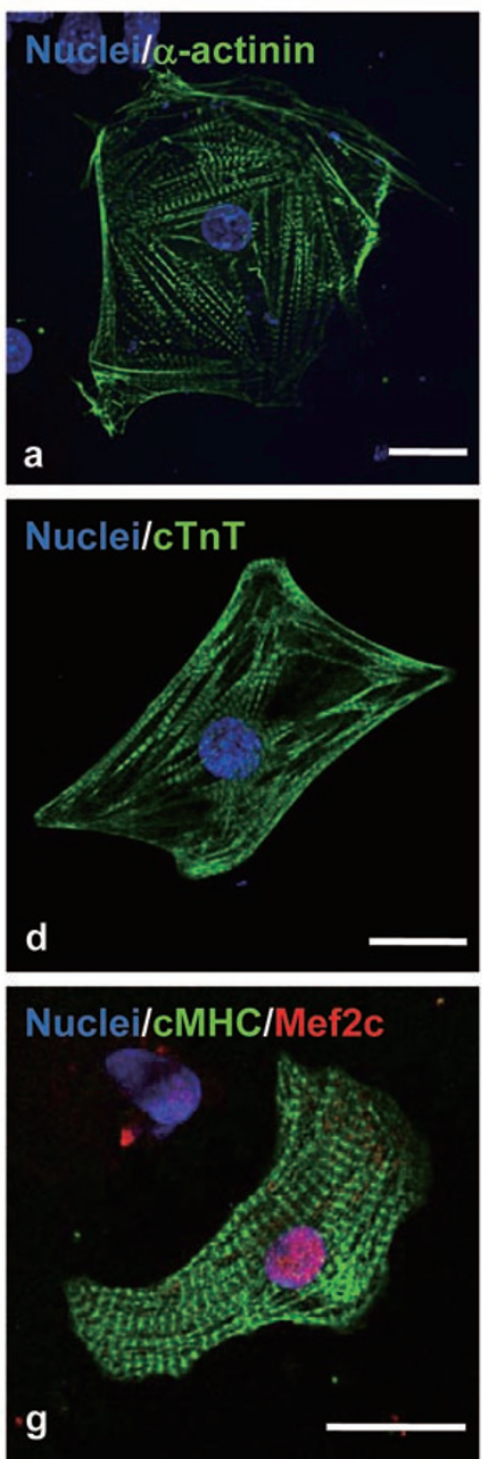

rFCMs


mESCMs


Figure 4 Comparison of sarcomeric structures of cardiomyocytes derived from rESCs and mESCs or isolated from rat fetal hearts by immunofluorescence staining of $\alpha$-actinin (upper panel), cTnT (middle panel), and cMHC and Mef2c (lower panel). Nuclei were stained with Hoechst 33258 (blue). Scale bars = $25 \mu \mathrm{m}$. rESCMs, rESC-derived cardiomyocytes; rFCMs, rat fetal cardiomyocytes; mESCMs, mESC-derived cardiomyocytes. 
A

Nodal

Atrial


rESCMs


\section{Ventricular}
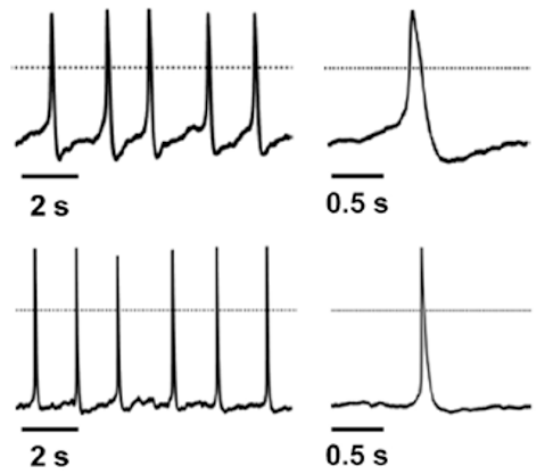

B

rESCMs

Control

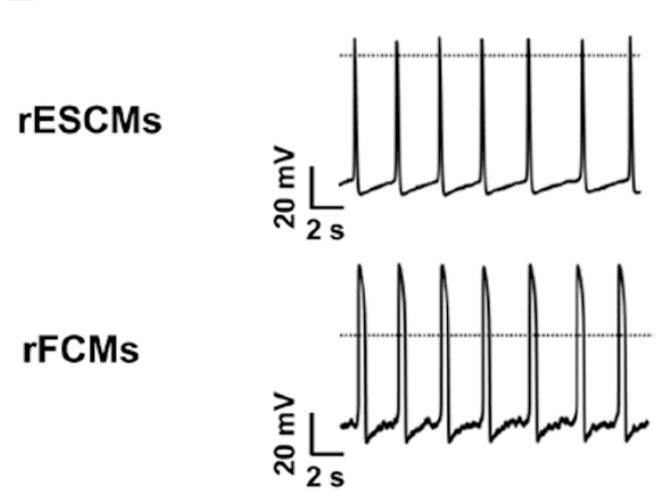

Iso
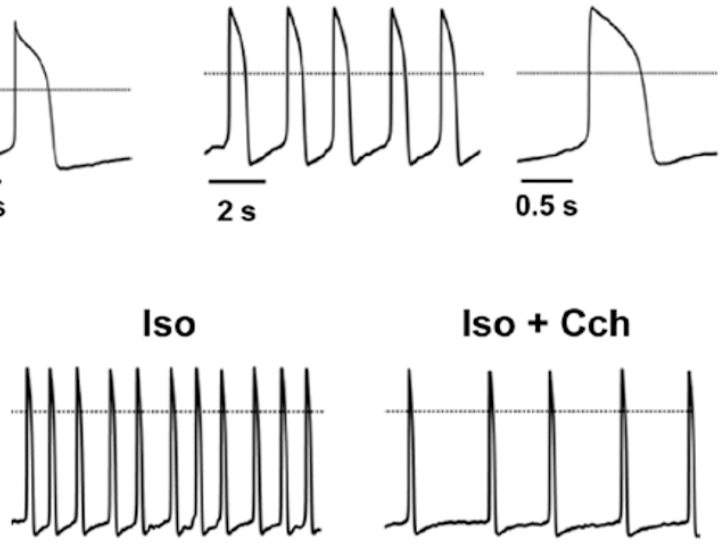

C


C

\section{Control}

$10 \mathrm{nM}$ Iso

$10 \mathrm{nM}$ Iso $+1 \mu \mathrm{M}$ Cch
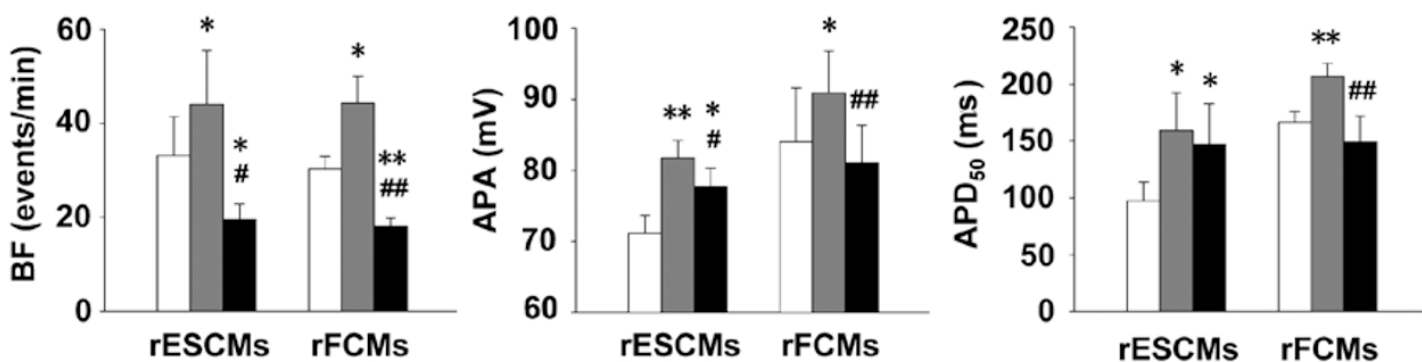

Figure 5 Action potentials recorded in rESC-derived cardiomyocytes (rESCMs) at differentiation day 16-18 and in cardiomyocytes isolated from rat fetal hearts ( $r F C M s)$ at the same developmental time. (A) Representative action potentials of $r E S C M s$ (left) and rFCMs (right) showing characteristics of nodal-like, atrial-like, and ventricular-like APs. Dotted lines indicate $0 \mathrm{mV}$. (B) Representative chronotropic effects of $\beta$-adrenergic agonist, isoproterenol (Iso, $10 \mathrm{nM}$ ), and muscarinic agonist, carbachol (Cch, $1 \mu \mathrm{M}$ ), in rESCMs and rFCMs. (C) Average beating frequency (BF), AP amplitude (APA), and AP duration at $50 \%$ of the amplitude $\left(\mathrm{APD}_{50}\right)$ in rESCMs and rFCMs before and during application of Iso and Cch. $n=5$ ( $\left.\mathrm{rESCMs}\right)$ and $n=7$ ( $\mathrm{rFCMs}$ ). ${ }^{*} P<0.05,{ }^{* *} P<0.01$ vs the corresponding control group; ${ }^{\#} P<0.05,{ }^{\# \#} P<0.01$ vs the corresponding Iso group. 
Table 1 Action potential properties of rESCMs and rFCMs

\begin{tabular}{llllllll}
\hline & $N$ & BF $($ events $/ \mathrm{min})$ & $\mathrm{APA}(\mathrm{mV})$ & $V_{\max }(\mathrm{V} / \mathrm{s})$ & $\mathrm{MDP}(\mathrm{mV})$ & $\mathrm{DD}^{(\mathrm{mV} / \mathrm{s})}$ & $\mathrm{APD}{ }_{50}(\mathrm{~ms})$ \\
\hline rESCMs & 32 & $39.2 \pm 5.5$ & $64.8 \pm 2.2$ & $2.7 \pm 0.2$ & $-46.1 \pm 1.5$ & $8.3 \pm 1.3$ & $124.8 \pm 11.9$ \\
$\mathrm{rFCMs}$ & 21 & $45.2 \pm 5.0$ & $79.2 \pm 5.0^{*}$ & $4.3 \pm 0.4^{* *}$ & $-45.5 \pm 2.8$ & $12.1 \pm 2.7$ & $193.6 \pm 9.9^{* *}$ \\
\hline
\end{tabular}

Data are presented as mean \pm SEM. rESCMs, rESCs-derived cardiomyocytes; rFCMs, rat fetal cardiomyocytes; AP, action potential; BF, beating frequency; APA, AP amplitude; $V_{\max }$, the maximum rate of rise of the AP; MDP, maximum diastolic potential; DD, rate of diastolic depolarization; $\mathrm{APD}_{50}$, AP duration at $50 \%$ of the amplitude; $* P<0.05, * * P<0.01$, compare with rESCMs group in Student's $t$-test.

action potentials (APs) in spontaneously beating single cardiomyocytes at day 16-18 differentiating rESCMs using whole-cell patch clamps. Based on the characteristics of main AP properties consisting of beating frequency (BF), AP amplitude (APA), the maximum rate of rise of the AP $\left(V_{\max }\right)$, the AP duration (APD), and prominence of phase 4 depolarization as previously described [29], three major types of APs, including nodal-like, atriallike, and ventricular-like AP, were detected in the rESCMs (Figure 5A). No significant differences of APs in BF, maximum diastolic potential (MDP), and rate of diastolic depolarization (DD) were detected between rESCMs and $\mathrm{rFCMs}$, whereas $\mathrm{rESCMs}$ showed lower APA, lower upstroke $V_{\max }$, and shorter AP duration at $50 \%$ of the amplitude $\left(\mathrm{APD}_{50}\right)$ compared with $\mathrm{rFCMs}$ (Table 1). These data demonstrate that the rESCMs have typical characteristics of APs of rFCMs, although some of the parameters show delayed development.

rESC-derived cardiomyocytes exhibit spontaneous rhythmic $\mathrm{Ca}^{2+}$ transients

Cyclic variations in the cytosolic-free $\mathrm{Ca}^{2+}$ concentration $\left(\left[\mathrm{Ca}^{2+}\right]_{\mathrm{i}}\right)$, named as $\mathrm{Ca}^{2+}$ transients, are essential events in the regulation of the contraction and relaxation of cardiomyocytes [22, 30]. We, therefore, measured spontaneous intracellular $\mathrm{Ca}^{2+}$ transients in day 16-18 cardiomyocytes derived from rESCs or isolated from rat fetal hearts. Spontaneous rhythmic $\mathrm{Ca}^{2+}$ transients were detected in rESCMs and rFCMs (Figure 6A). $\mathrm{Ca}^{2+}$ transients recorded from rESCMs showed similar BF, amplitude, upstroke $V_{\max }$, and decay rates as compared with those from $\mathrm{rFCMs}$ at comparable developmental stages $(P$ $>0.05$, Figure $6 \mathrm{~B}$ ), indicating that $\mathrm{rESCMs}$ have similar functional coupling as those observed in the rFCMs.

\section{Presence of $\beta$-adrenergic and muscarinic responses in rESCMs}

Intact responses to hormones and transmitters of the central nervous system are one of the most critical characteristics of normal cardiomyocytes. We thus studied the response of rESCMs to $\beta$-adrenergic and muscarinic stimulations that activate critical signaling pathways in cardiomyocytes and subsequently influence cardiac APs and $\mathrm{Ca}^{2+}$ transients. Application of $10 \mathrm{nM} \beta$-adrenergic agonist, isoproterenol (Iso), comparably increased the frequency, amplitude, and $\mathrm{APD}_{50}$ of APs in rESCMs compared with those observed in $\mathrm{rFCMs}(P<0.05, n$ $=5$ in rESCMs and 7 in rFCMs, Figure $5 \mathrm{~B}$ and $5 \mathrm{C}$, respectively). Iso at $10 \mathrm{nM}$ also significantly increased the frequency, amplitude, and upstroke $V_{\max }$ of $\mathrm{Ca}^{2+}$ transients in rESCMs and in rFCMs (Figure 6A and 6B), whereas there was no significant change in the decay rate of $\mathrm{Ca}^{2+}$ transients in rESCMs $(P>0.05$, Figure 6B, bottom right panel). In addition, subsequent application of $1 \mu \mathrm{M}$ muscarinic agonist, carbachol (Cch), not only blocked Iso-increased beating frequency of the APs but also had negative chronotropic effects in both rESCMs and $\mathrm{rFCMs}$. Cch also inhibited Iso-increased APA but not the $\mathrm{APD}_{50}$ of APs in rESCMs, whereas the rFCMs were more sensitive to $\mathrm{Cch}$ stimuli (Figure $5 \mathrm{~B}$ and $5 \mathrm{C}$ ). These data overall indicate that coupled $\beta$-adrenergic and muscarinic signaling cascades, as well as their associated intracellular signaling partners, are present and functional in the cardiomyocytes derived from rESCs, although the rFCMs seem relatively more mature compared with the rESCMs.

\section{Discussion}

Recent reports have demonstrated that pluripotent ESCs or ESC-like lines can be generated from rats [6$9,11,12,31]$. However, the difficulty of differentiating these cells in vitro represents an obstacle to achieving their full values. In the present study, we report that: (i) a stable in vitro differentiation system for rESCs is successfully established through which rESCs are capable of forming EBs efficiently and differentiating into all three germ layers; (ii) rESCs cultivated under such conditions can differentiate in vitro into the derivatives from mesoderm as represented by cardiomyocytes; and (iii) rESCMs show structural and functional properties comparable with the early-stage rFCMs. The established differentiation system will greatly contribute to the understanding of rESC biology by dissecting the process at the cellular level and to the development of cardiac-regenerative medicines. 
A
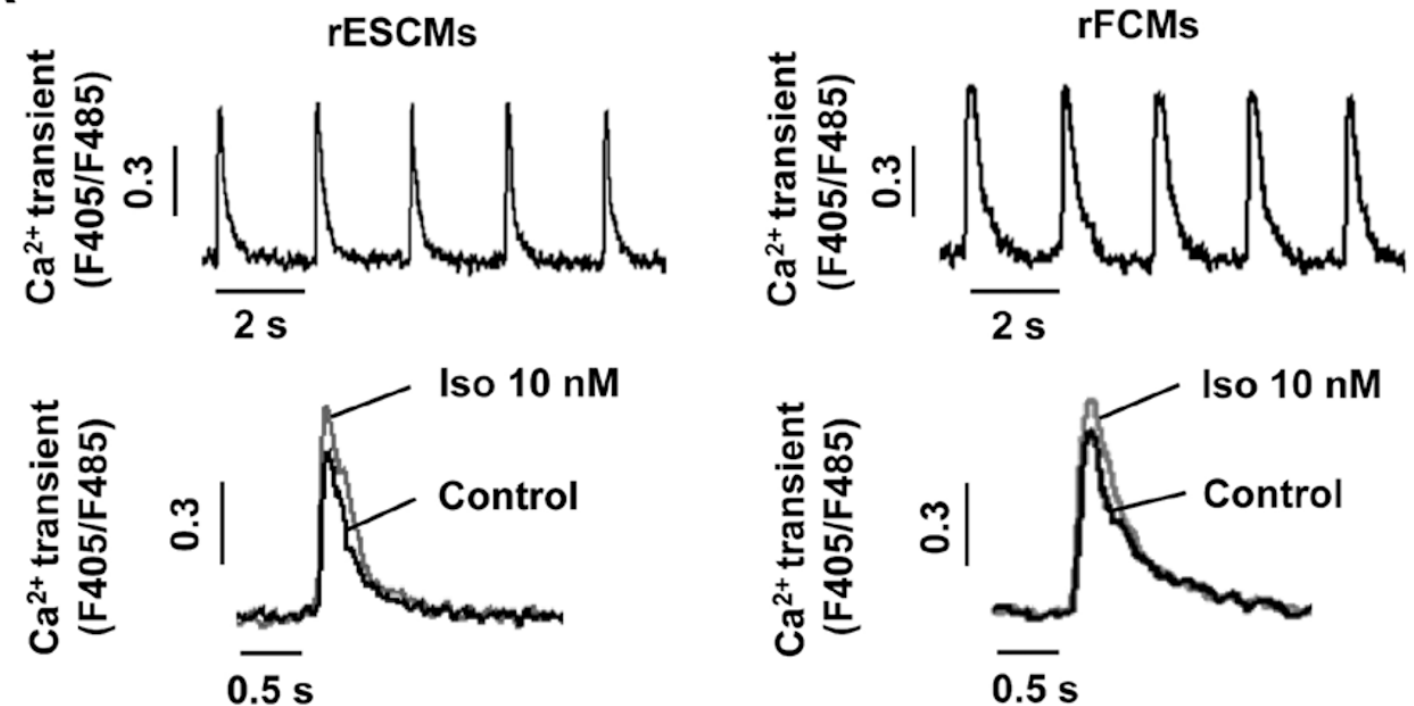

B
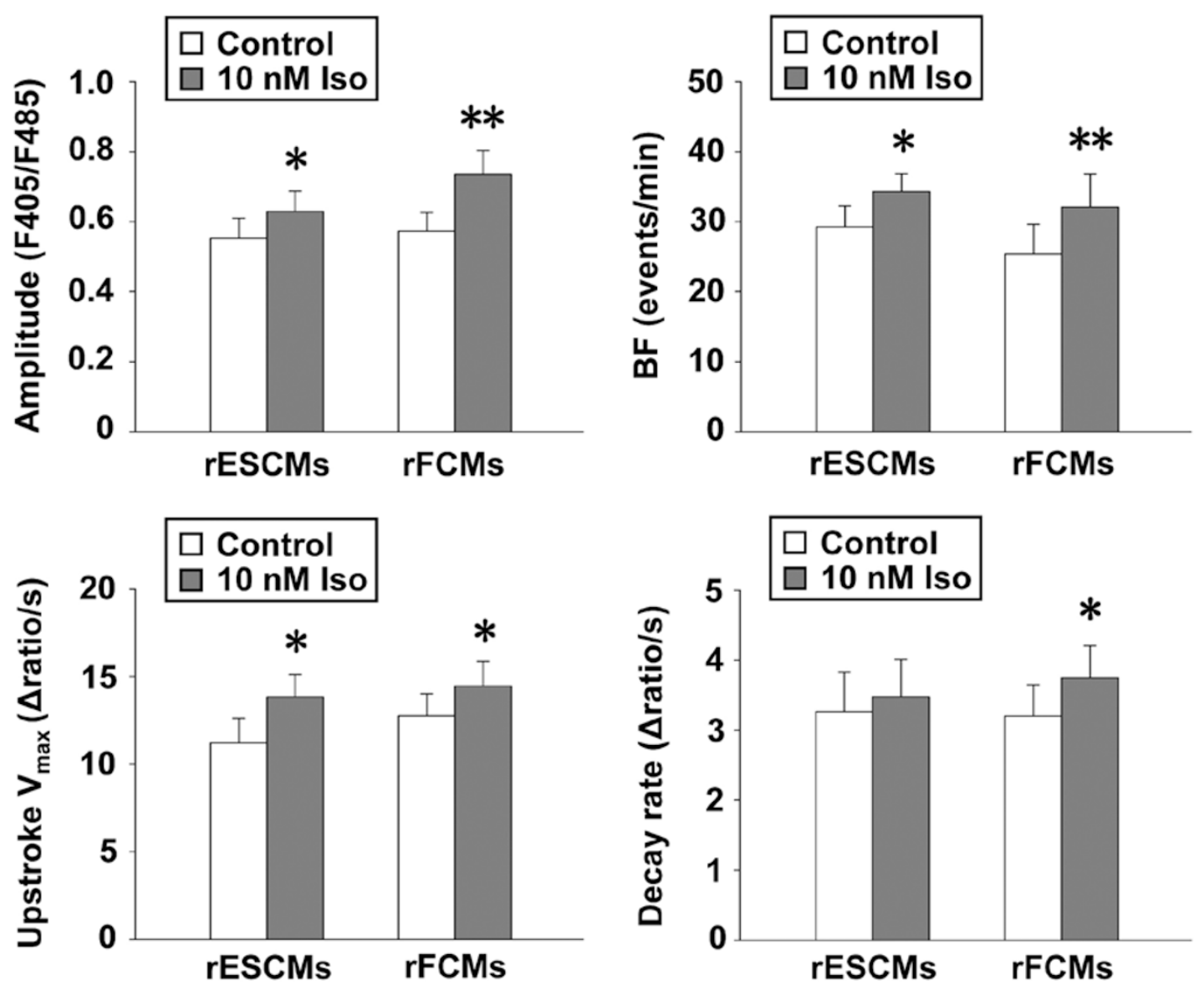

Figure 6 Dynamics of spontaneous intracellular $\mathrm{Ca}^{2+}$ transients in rESCMs and rFCMs at differentiation/embryonic day 16-18. (A) Representative tracings of spontaneous rhythmic $\mathrm{Ca}^{2+}$ transients with (lower panel) and without (upper panel) $\beta$-adrenergic stimulation by its agonist, isoproterenol (Iso, $10 \mathrm{nM})$. (B) Effects of Iso on the amplitude, BF, upstroke $V_{\max }$, and decay rate of $\mathrm{Ca}^{2+}$ transients in rESCMs and rFCMs; $n=7$ each. ${ }^{*} P<0.05,{ }^{* *} P<0.01$ vs the corresponding control group. 
ESCs are unique tools not only for studying mammalian development, regenerative medicine, and diseases $[15,19,20,32,33]$ but also for pharmacological testing and drug screening in the culture dishes [34-36]. As physiological regulation and pharmacological response in rats are relevant to humans [1-3], the establishment of bona fide rESC lines is extremely useful for those studies. However, efficiently differentiating these cells into derivatives of the three germ layers in vitro is a critical prerequisite in achieving their full potential. The formation of EBs is the primary way to induce ESC differentiation in vitro $[15,37,38]$, whereas the proper approach for EB formation varies from species to species [39]. In previous studies, researchers have optimized the EB formation method of ESCs from mouse and human [19, 40-42], but this method does not work well in rESCs as a majority of the cells die when forming EBs $[7,11]$. In this study, we have developed a system for the efficient formation of EBs from rESCs by harnessing the application of signal inhibitors and hanging-drop technology. Compared with the protocol reported previously [7], we have made the following changes: (i) we used the classical hanging-drop method so that the size of EBs could be controlled and the formation of EBs is highly uniform; (ii) we modified the EB cultivation system by direct induction of EB formation in the rEFM containing serum and 2i, CHIR99021 and PD0325901, instead of the protocol of sequential application of $3 \mathrm{i}$ and CM; (iii) we added Rho-associated kinase inhibitor, Y-27632, to suppress apoptosis of ESCs [23]; and (iv) we decreased the concentration of $2 \mathrm{i}$ to minimize their potentially negative effects on rESC differentiation. Our data demonstrate that these modifications are critical for rESCs to survive, form uniform EBs efficiently, and differentiate into three embryonic germ layers steadily (Figure 2).

To exclude the possibility that the acute death of rESCs when initiating differentiation is caused by the relative harsh dissociation by trypsin, we tried gentler enzymes including collagenase IV, dispase, or TrypLE instead of Trypsin. We found a similar requirement of rEFM for EB formation from these enzyme-treated cells compared with the trypsin-dissociated ones as the majority of cells also died when DM was used and wellshaped EBs were only observed in rEFM cultivation (data not shown). These results indicate that the acute death of rESCs appears not to be caused by trypsin dissociationinduced apoptosis but by the impetuous change of culture environment upon the initiation of differentiation process. This view is also supported by the fact that no obvious cell death was observed when undifferentiated rESCs were tripsinized and passaged. Previous reports have shown that U- [40] and V-shaped plate systems [42] facilitate the formation of homogenous EBs from human ESCs. We observed a similar requirement of rEFM for EB formation from the rESCs either with U- and V-shaped systems (Supplementary information, Figure S3A) or with the hanging-drop method that we used (Figure 2A). Consistently, a comparable cardiac-differentiation efficiency is observed between the hanging-drop method we used and the use of U- as well as V-shaped plate system (Supplementary information, Figure S3B). Moreover, the impact of rEFM on cardiac differentiation of rESCs was also highlighted by the fact that EBs generated in rEFM differentiated into cardiomyocytes more efficiently based on our systematic tests (Supplementary information, Figure S2). Taken together, our results suggest that the EB formation of rESCs needs a specific environment to maintain sufficient cell survival during the initiation of differentiation process.

Molecular analyses demonstrate that cardiogenesis of rESCs include a sequential expression of key transcripts for mesoderm, cardiovascular and cardiac precursors, and cardiomyocytes (Figure 3), which is consistent with the process of mammalian heart development in vivo and in vitro mESC models [27, 28, 43-45]. Structural investigation demonstrates that $\mathrm{rESCMs}$ are comparable with the ones from the rat embryonic heart and mESCs (Figure 4). Moreover, the rESCs cultivated under such conditions can stably differentiate into contracting cardiomyocytes from differentiation day 9 (Figure 3C), although the efficiency of cardiac differentiation of rESCs (about $40 \%$ ) is relatively low compared with that of mESCs $(>80 \%$, under suitable conditions) [19] based on the percentage of EBs with contracting clusters. Such variations may represent differences between the species and the cell lines, some currently undetermined factors required in the in vitro differentiation system, or native deficiency in cell adhesion of rESCs $[6,7,13]$. Therefore, the in vitro differentiation system of rESCs into cardiomyocytes needs to be optimized in the future by testing growth factors, supporting stroma, and different serum content, among other possibilities.

Electrophysiological analyses by means of APs reveal that rESCMs contain at least three typical cardiac cells, i.e., nodal, atrial, and ventricular cells as observed from cardiomyocytes derived from mESCs [46] and human ESCs [29]. Moreover, rESCMs display properties of APs and spontaneous intracellular $\mathrm{Ca}^{2+}$ transients comparable with $\mathrm{rFCMs}$ (Figures 5 and 6) and $\mathrm{mESCMs}$ that we observed previously [22, 47, 48]. Furthermore, the presence of similar responses to $\beta$-adrenergic and muscarinic stimulations in the rESCMs as those found in the rFCMs (Figures 5 and 6) demonstrates that rESCMs have already established critical signal-transduction pathways 
and all components of normal cardiac excitation-contraction coupling.

In conclusion, we successfully established a stable in vitro differentiation system for $\mathrm{rESCs}$ through which rESCs differentiate into three germ layers and generate functional cardiomyocytes. Given the outstanding potential demonstrated by mESCs, the establishment of this in vitro differentiation system may have a significant impact on the study of commitment and differentiation of rat embryonic tissues under in vitro conditions. Using this system, the spontaneously contracting clusters were, for the first time to our knowledge, demonstrated to possess structural and functional properties consistent with the phenotype of rFCMs. Thus, this in vitro cardiomyocytedifferentiation system may be a powerful research tool in several fields such as study of early cardiomyogenesis, pharmacological and toxicological testing, and cell therapy.

\section{Materials and Methods}

\section{rESC derivation and propagation}

Rat blastocysts from inbred strain DA were gently flushed out from the uteruses of E4.5 pregnant rats with DMEM medium. After the removal of the zona with Pronase (Sigma, St Louis, MO, USA), a single blastocyst was transferred onto 24-well plates and cultured in serum-free N2B27 medium supplemented with 2i (MEK inhibitor: PD0325901, $0.4 \mu \mathrm{M}$; and GSK3 inhibitor: CHIR99021, $3 \mu \mathrm{M}$ ) on mitomycin C-inactivated MEF feeder layers modified from the method reported previously [6, 7]. N2B27 medium was prepared as described previously [49]. PD0325901 and CHIR99021 were purchased from Stemgent Corporation (San Diego, CA, USA). After 5-7 days, the outgrowths of blastocysts were disaggregated and replated in the same MEF-2 $\mathrm{i}$ conditions. Established rESC lines DA8-16 and DA8-21 were routinely maintained in MEF-2i conditions and the medium was changed daily. rESCs were passaged by dissociation with $0.05 \%$ trypsin (Invitrogen/Gibco, Carlsbad, CA, USA) and transferred onto fresh MEF feeder cells every 2-3 days. As both cell lines showed similar characteristics, only one of them (cell line DA8-16) was used for analysis in this study unless indicated. Undifferentiated R1 mESCs were cultured in the medium containing 15\% FBS (Hyclone, Logan, UT, USA) on mitomycin C-inactivated MEF cells in the presence of LIF (Millipore, Billerica, MA, USA, $1000 \mathrm{U} / \mathrm{ml}$ ) as previously described [47]. All cultivation medium and other substances for cell cultures were purchased from Invitrogen/Gibco BRL if not indicated.

\section{Teratoma formation}

The rESCs were trypsinized into single cells and injected intramuscularly into NOD/SCID mice (approximately $1 \times 10^{7}$ cells per site). Two to three mice were injected for each cell line. After 30 days, tumors were processed for hematoxylin and eosin staining. All animal experiments were conducted in accordance with the Guide for the Care and Use of Animals for Research Purposes and were approved by the SIBS Animal Care Committee.

\section{Chimera generation}

Rat blastocysts at E4.5 days post coitum (dpc) were collected by noon on the day of injection and cultured for $2-3 \mathrm{~h}$ in $10 \% \mathrm{FBS}$ DMEM medium to ensure cavitation. rESCs were disaggregated and 15-20 cells were injected into the blastocyst cavities. Injected embryos were transferred into uteruses of pseudo pregnant SD females.

\section{EB formation and differentiation of rESCs}

The cultivation media used to test the EB formation and differentiation of rESCs are indicated as follows: (i) DM, DMEM/ F12 supplemented with $10 \%$ FBS, $100 \mu \mathrm{M} \beta$-mercaptoethanol (Sigma), 1\% NEAA, and $2 \mathrm{mM}$ Glutamine; (ii) CM, the DM containing 20\% FBS were conditioned and collected from MEF cells, then mixed at 1:1 with N2B27 medium to get the final concentration of FBS at $10 \%$; and (iii) rEFM, the CM added with $10 \mu \mathrm{M}$ of Y27632 (Calbiochem, Gibbstown, NJ, USA) and 2i (MEK inhibitor: PD0325901, $0.1 \mu \mathrm{M}$; and GSK3 inhibitor: CHIR99021, 0.75 $\mu \mathrm{M})$. Before EB formation, rESCs were trypsinized and plated onto gelatin-coated culture dishes in the rEFM for 30-40 $\mathrm{min}$ to remove MEF cells. Next, the non-adherent cell suspension consisting mainly of $\mathrm{rESCs}$ was diluted into $10^{5}$ cells $/ \mathrm{ml}$ and aliquoted into $30 \mu \mathrm{l}$ drops on the lid of $100 \mathrm{~mm}$ petri dishes following the standard hanging-drop method $[18,19]$. The EBs were harvested 2 days later and then transferred onto ultralow attachment plates (Corning, Canton, NY, USA) for 4 days of suspension culture and $2 \mathrm{i}$ were eliminated at day 3 (Figure 2D). After 6 days of differentiation, the EBs were seeded onto matrigel-coated plates (BD Biosciences, Bedford, MA, USA) for adhered culture and examinations.

\section{$R T-P C R$}

Total RNA was isolated from the cells of rat fetal heart using an RNeasy Plus Mini Kit (QIAGEN, Valencia, CA, USA) following the manufacturer's instructions and treated with DNAse I (Promega, Madison, WI, USA) for $15 \mathrm{~min}$ to eliminate the potential contamination of genomic DNA. Total RNA $(1 \mu \mathrm{g})$ was transcribed into cDNA using oligo (dT) primer and ReverTra Ace reverse transcriptase (Toyobo, Osaka, Japan). PCR was carried out using Taq DNA Polymerase (Takara, Shiga, Japan). The PCR primers of interest are listed in Supplementary information, Table S1. Glyceraldehydes 3-phosphate dehydrogenase (Gapdh) was used as endogenous control, and samples without reverse transcription were used as negative control.

\section{$Q-P C R$}

Q-PCR was performed and analyzed by kinetic real-time PCR using the ABI PRISM 7900 system (Applied Biosystems, Foster City, CA, USA) with SYBR Green Realtime PCR Master Mix plus (Toyobo) for relative quantification of the indicated genes. The transcript of Gapdh was used for internal normalization. The Q-PCR primers are listed in Supplementary information, Table S2.

\section{Apoptosis assays and flow cytometry analysis}

Annexin V-PI double stainings were used to evaluate apoptosis and necrosis levels of the EBs formed in representative conditions. After 2 days of differentiation in suspension, EBs were digested into single cells using non-enzyme dissociation buffer (Invitrogen) and stained with $0.5 \mu \mathrm{g} / \mathrm{ml}$ PI and APC-labeled Annexin V anti- 
body (BD Biosciences) at a 1:20 dilution according to the manufacturer's instructions.

To investigate the percentage of cardiomyocytes in the total cell population by using our protocol, EBs after 15 days of differentiation were harvested and dissociated. To detect the intracellular antigen, cells were fixed and permeabilized by Foxp3 Staining Buffer Set (eBioscience, San Diego, CA, USA), blocked by $10 \%$ normal goat serum (Vector Laboratories, Burlingame, CA, USA) and incubated with primary antibody of cTnT (1:100; Abcam, La Jolla, CA, USA) or a mouse IgG1 antibody (BD Bioscience) as an isotype control. PE-conjugated anti-mouse Ig multiple antibody (BD Bioscience) were used as secondary antibody.

Cells were analyzed and quantified by flow cytometry (FACStar Plus Flow Cytometer, Becton-Dickson).

Isolation of single cardiomyocytes from mouse/rat beating EBs or fetal hearts

Methods to prepare isolated cardiomyocytes for immunostaining and electrophysiology analyses were described previously [47]. Briefly, E15.5 embryos and differentiation day-matched beating EBs were dissected and dissociated by trypsin and collagenase B (Invitrogen). The isolated cells were seeded on sterile, laminincoated glass coverslips and incubated for $12-60 \mathrm{~h}$ prior to the experiments.

\section{Immunocytochemical staining analysis}

ALP activity was determined by staining with an ALP substrate kit III (Vector Laboratories) according to the manufacturer's instructions. Undifferentiated ESCs or dissociated EBs were seeded on laminin-coated slides and cultured for 2-3 days to allow tough attachment. Cells were fixed in 4\% paraformaldehyde for $20 \mathrm{~min}$ and permeabilized in $0.3 \%$ Triton X-100 (Sigma) for $30 \mathrm{~min}$ at room temperature. After a single wash of PBS, cells were blocked in $10 \%$ normal goat serum supplemented with $0.1 \%$ Triton X-100 at room temperature for $1 \mathrm{~h}$, then incubated with primary antibodies against Oct4 (1:200), Nanog (1:100; Calbiochem), SSEA1 (1:250; Millipore), Sox17 (1:100; R\&D Systems, Minneapolis, MN, USA), Flk1 (1:200; BD Biosciences), Map2 (1:200; Abcam), $\alpha$-actinin (1:200; Sigma), cTnT (1:100; Abcam), cMHC (1:100; Abcam), and Mef2c (1:100; Cell Signaling, Beverly, MA, USA) in $4{ }^{\circ} \mathrm{C}$ overnight and detected by DyLight 488 - or DyLight 549-conjugated secondary antibodies (Jackson ImmunoResearch, West Grove, PA, USA). Nuclei were stained with Hoechst 33258 (Sigma) and staining without primary antibodies was used to be a negative control. A Leica TCS SP2 confocal laser scanning microscope was used for slide observation and image capture.

\section{Whole-cell patch clamp}

Whole-cell patch clamps using the EPC-10 amplifier (Heka Electronics, Bellmore, NY, USA) in current-clamp mode were used to record APs in spontaneously beating single cardiomyocytes as described earlier [48]. For AP recording, the pipette electrode (2-6 M $\Omega$ ) was filled with a solution containing (mmol/l): 50 $\mathrm{KCl}, 80 \mathrm{~K}$-Aspartate, $5 \mathrm{MgCl}_{2}$, 5 EGTA, 10 Hepes, and $5 \mathrm{Na}_{2} \mathrm{ATP}$ (pH 7.2 adjusted with $\mathrm{KOH}$ ). The extracellular bathing solution contained (mmol/1): $135.0 \mathrm{NaCl}, 5.4 \mathrm{KCl}, 1.8 \mathrm{CaCl}_{2}, 1.0 \mathrm{MgCl}_{2}$, 10.0 glucose, and 10.0 HEPES ( $\mathrm{pH} 7.4$, adjusted with $\mathrm{NaOH}$ ). The glass coverslips containing the cells were placed into a temperature-controlled $\left(35^{\circ} \mathrm{C}\right)$ recording chamber and perfused continu- ously with extracellular solution.

\section{Measurement of $\mathrm{Ca}^{2+}$ transients}

Isolated $\mathrm{rESCMs}$ or $\mathrm{rFCMs}$ were loaded with $5 \mathrm{~mol} / \mathrm{l}$ indo-1 AM and $0.45 \%$ pluronic F-127 (Molecular Probes, Eugene, OR, USA) for $10 \mathrm{~min}$ at room temperature [47, 48]. Loaded cells were washed with a solution containing (mmol/l): $135.0 \mathrm{NaCl}, 5.4$ $\mathrm{KCl}, 1.8 \mathrm{CaCl}_{2}, 1.0 \mathrm{MgCl}_{2}, 10.0$ glucose, and 10.0 HEPES (pH 7.4 at $35{ }^{\circ} \mathrm{C}$ ). Fluorescence signals of Indo- 1 were detected by a Fluorescence/Contractility System (IonOptix, Milton, MA, USA). Fluorescence signals were excited at $360 \pm 5 \mathrm{~nm}$ with an ultraviolet light source, and the emitted fluorescence was measured at 405 and $485 \mathrm{~nm}$ using two photomultipliers attached to an inverted microscope (Olympus, Tokyo, Japan). After the subtraction of background fluorescence, the ratio of fluorescence (R) emitted at 405 and $485 \mathrm{~nm}$ was recorded [50] and analyzed with the IonWizard 6.0 software (IonOptix).

\section{Statistical analysis}

Data are expressed as mean \pm SEM. Statistical significance of differences was estimated by one way ANOVA or Student's $t$-test. $P<0.05$ was considered significant.

\section{Acknowledgments}

The study was supported by grants from the State Major Research Program of China (2007CB947100, 2010CB945603, and 2011CB965301), National Science and Technology Major Project (2009ZX09503-024), Knowledge Innovation Program of CAS (KSCX2-YW-R-233, KSCX1-YW-R-46), Strategic Priority Research Program of CAS (XDA01030000), and the Ministry of Agriculture (2009ZX08010-016B). We thank Zhongyan Chen for performing fetal rat heart isolation, $\mathrm{Lan} \mathrm{Wu}$ for technique assistance in the measurement of Ca2+ transients, Lei Qian and Jihui Zhang for performing rESCs culture, and Dr Ying Jin for kindly providing the Oct4 antibody.

\section{References}

1 Aitman TJ, Critser JK, Cuppen E, et al. Progress and prospects in rat genetics: a community view. Nat Genet 2008; 40:516-522.

2 Jacob HJ, Kwitek AE. Rat genetics: attaching physiology and pharmacology to the genome. Nat Rev Genet 2002; 3:33-42.

3 Jacob HJ. Functional genomics and rat models. Genome Res 1999; 9:1013-1016.

4 Doggrell SA, Brown L. Rat models of hypertension, cardiac hypertrophy and failure. Cardiovasc Res 1998; 39:89-105.

5 Bader M. Rat models of cardiovascular diseases. Methods Mol Biol 2010; 597:403-414.

6 Buehr M, Meek S, Blair K, et al. Capture of authentic embryonic stem cells from rat blastocysts. Cell 2008; 135:12871298.

7 Li P, Tong C, Mehrian-Shai R, et al. Germline competent embryonic stem cells derived from rat blastocysts. Cell 2008; 135:1299-1310.

8 Hirabayashi M, Kato M, Kobayashi T, et al. Establishment of rat embryonic stem cell lines that can participate in germline chimerae at high efficiency. Mol Reprod Dev 2010; 77:94. 
9 Kawamata M, Ochiya T. Establishment of embryonic stem cells from rat blastocysts. Methods Mol Biol 2010; 597:169177.

10 Demers SP, Smith LC. Derivation, culture, and in vivo developmental capacity of embryonic cell lines from rat blastocysts. Methods Mol Biol 2010; 597:179-188.

11 Zhao X, Lv Z, Liu L, et al. Derivation of embryonic stem cells from Brown Norway rats blastocysts. J Genet Genomics 2010; 37:467-473.

12 Kawamata M, Ochiya T. Generation of genetically modified rats from embryonic stem cells. Proc Natl Acad Sci USA 2010; 107:14223-14228.

13 Dolgin E. The knockout rat pack. Nat Med 2010; 16:254-257.

14 Tong C, Li P, Wu NL, Yan Y, Ying QL. Production of p53 gene knockout rats by homologous recombination in embryonic stem cells. Nature 2010; 467:211-213.

15 Keller GM. In vitro differentiation of embryonic stem cells. Curr Opin Cell Biol 1995; 7:862-869.

16 Smith AG. Embryo-derived stem cells: of mice and men. Annu Rev Cell Dev Biol 2001; 17:435-462.

17 Keller G. Embryonic stem cell differentiation: emergence of a new era in biology and medicine. Genes Dev 2005; 19:11291155.

18 Wobus AM, Guan K, Yang HT, Boheler KR. Embryonic stem cells as a model to study cardiac, skeletal muscle, and vascular smooth muscle cell differentiation. Methods Mol Biol 2002; 185:127-156.

19 Boheler KR, Czyz J, Tweedie D, et al. Differentiation of pluripotent embryonic stem cells into cardiomyocytes. Circ Res 2002; 91:189-201.

20 Winkler J, Hescheler J, Sachinidis A. Embryonic stem cells for basic research and potential clinical applications in cardiology. Biochim Biophys Acta 2005; 1740:240-248.

21 Puceat M. Protocols for cardiac differentiation of embryonic stem cells. Methods 2008; 45:168-171.

22 Fu JD, Yu HM, Wang R, Liang J, Yang HT. Developmental regulation of intracellular calcium transients during cardiomyocyte differentiation of mouse embryonic stem cells. Acta Pharmacol Sin 2006; 27:901-910.

23 Watanabe K, Ueno M, Kamiya D, et al. A ROCK inhibitor permits survival of dissociated human embryonic stem cells. Nat Biotechnol 2007; 25:681-686.

24 Wobus AM. Potential of embryonic stem cells. Mol Aspects Med 2001; 22:149-164.

25 Kattman SJ, Adler ED, Keller GM. Specification of multipotential cardiovascular progenitor cells during embryonic stem cell differentiation and embryonic development. Trends Cardiovasc Med 2007; 17:240-246.

26 Wu SM, Chien KR, Mummery C. Origins and fates of cardiovascular progenitor cells. Cell 2008; 132:537-543.

27 Martin-Puig S, Wang Z, Chien KR. Lives of a heart cell: tracing the origins of cardiac progenitors. Cell Stem Cell 2008; 2:320-231.

28 Christoforou N, Miller RA, Hill CM, et al. Mouse ES cellderived cardiac precursor cells are multipotent and facilitate identification of novel cardiac genes. J Clin Invest 2008; 118:894-903.

29 He JQ, Ma Y, Lee Y, Thomson JA, Kamp TJ. Human embryonic stem cells develop into multiple types of cardiac myo- cytes: action potential characterization. Circ Res 2003; 93:3239.

30 Bers DM. Sarcoplasmic reticulum Ca release in intact ventricular myocytes. Front Biosci 2002; 7:d1697-d1711.

31 Li C, Yang Y, Gu J, Ma Y, Jin Y. Derivation and transcriptional profiling analysis of pluripotent stem cell lines from rat blastocysts. Cell Res 2009; 19:173-186.

32 Murry CE, Keller G. Differentiation of embryonic stem cells to clinically relevant populations: lessons from embryonic development. Cell 2008; 132:661-680.

33 Rossant J. Stem cells and early lineage development. Cell 2008; 132:527-531.

34 Wartenberg M, Gunther J, Hescheler J, Sauer H. The embryoid body as a novel in vitro assay system for antiangiogenic agents. Lab Invest 1998; 78:1301-1314.

35 Harding SE, Ali NN, Brito-Martins M, Gorelik J. The human embryonic stem cell-derived cardiomyocyte as a pharmacological model. Pharmacol Ther 2007; 113:341-353.

36 Winkler J, Sotiriadou I, Chen S, Hescheler J, Sachinidis A. The potential of embryonic stem cells combined with -omics technologies as model systems for toxicology. Curr Med Chem 2009; 16:4814-4827.

37 Desbaillets I, Ziegler U, Groscurth P, Gassmann M. Embryoid bodies: an in vitro model of mouse embryogenesis. Exp Physiol 2000; 85:645-651.

38 Itskovitz-Eldor J, Schuldiner M, Karsenti D, et al. Differentiation of human embryonic stem cells into embryoid bodies compromising the three embryonic germ layers. Mol Med 2000; 6:88-95.

39 Kurosawa H. Methods for inducing embryoid body formation: in vitro differentiation system of embryonic stem cells. $J$ Biosci Bioeng 2007; 103:389-398.

$40 \mathrm{Ng}$ ES, Davis RP, Azzola L, Stanley EG, Elefanty AG. Forced aggregation of defined numbers of human embryonic stem cells into embryoid bodies fosters robust, reproducible hematopoietic differentiation. Blood 2005; 106:1601-1603.

41 Wang X, Wei G, Yu W, et al. Scalable producing embryoid bodies by rotary cell culture system and constructing engineered cardiac tissue with ES-derived cardiomyocytes in vitro. Biotechnol Prog 2006; 22:811-818.

42 Burridge PW, Anderson D, Priddle H, et al. Improved human embryonic stem cell embryoid body homogeneity and cardiomyocyte differentiation from a novel V-96 plate aggregation system highlights interline variability. Stem Cells 2007; 25:929-938.

43 Parmacek MS, Epstein JA. Pursuing cardiac progenitors: regeneration redux. Cell 2005; 120:295-298.

44 Yang L, Soonpaa MH, Adler ED, et al. Human cardiovascular progenitor cells develop from a KDR+ embryonic-stem-cellderived population. Nature 2008; 453:524-528.

$45 \mathrm{Bu} \mathrm{L}$, Jiang X, Martin-Puig S, et al. Human ISL1 heart progenitors generate diverse multipotent cardiovascular cell lineages. Nature 2009; 460:113-117.

46 Hescheler J, Fleischmann BK, Lentini S, et al. Embryonic stem cells: a model to study structural and functional properties in cardiomyogenesis. Cardiovasc Res 1997; 36:149-162.

$47 \mathrm{Fu}$ JD, Li J, Tweedie D, et al. Crucial role of the sarcoplasmic reticulum in the developmental regulation of $\mathrm{Ca} 2+$ transients and contraction in cardiomyocytes derived from embryonic 
stem cells. FASEB J 2006; 20:181-183.

48 Yang HT, Tweedie D, Wang S, et al. The ryanodine receptor modulates the spontaneous beating rate of cardiomyocytes during development. Proc Natl Acad Sci USA 2002; 99:92259230.

49 Nichols J, Ying QL. Derivation and propagation of embryonic stem cells in serum- and feeder-free culture. Methods Mol Biol 2006; 329:91-98.

50 Grynkiewicz G, Poenie M, Tsien RY. A new generation of $\mathrm{Ca} 2+$ indicators with greatly improved fluorescence properties. J Biol Chem 1985; 260:3440-3450.

(Supplementary information is linked to the online version of the paper on the Cell Research website.) 